R. ZHAO

KODAI MATH. J.

15 (1992), 221-229

\title{
THE CHARACTERISTICS OF BMOA ON RIEMANN SURFACES
}

\author{
BY ZHAO RUHAN
}

\begin{abstract}
In this paper we give a John-Nirenberg type theorem for BMOA on general open Riemann surfaces. Using $\mathrm{Ba}$ spaces we give a new characteristic for BMOA on Riemann surfaces in this paper too.
\end{abstract}

\section{Introduction.}

In [7], T.A. Metzger asked whether the John-Nirenberg theorem for BMOA on the unit disk is true on Riemann surfaces. We have given a positive answer for compact bordered Riemann surfaces in [4]. In this paper we will give a John-Nirenberg type theorem for BMOA on general open Riemann surfaces. Some new characteristics of BMOA on Riemann surfaces will be given in this paper too.

\section{John-Nirenberg type theorem for BMOA on Riemann surfaces.}

Let $R$ be an open Riemann surface which possesses a Green's function, i.e., $R \notin O_{G}$. Let $G_{R}(w, a)$ be the Green's function of $R$ with logarithmic singularity at $a \in R$. We firstly give an important lemma as follows:

LEMMA 2.1. Let $R_{1} \subset R_{2} \subset \cdots \subset R_{k} \rightarrow R$ be an exhaustion of the Rremann surface $R$, where $R_{k}$ are compact bordered Riemann surfaces $(1 \leqq k<\infty) . \quad F$ is an analytic function on $R$. Let the least harmonic majorant of the subharmonic function $|F(w)|^{p}$ on $R\left(\right.$ or $\left.R_{k}\right)$ be $H(w)$ (or $\left.H_{k}(w)\right)$. Then

$$
H(w)=\sup _{k \geq 1} H_{k}(w)=\lim _{k \rightarrow \infty} H_{k}(w) .
$$

If $F(w)$ has no harmonic majorant on $R$ (or $\left.R_{k}\right)$ we denote $H(w)=\infty\left(\right.$ or $H_{k}(w)$ $=\infty)$.

Proof. It is easy to verify that $\left\{H_{k}(w)\right\}$ is an increasing sequence. By Hanack theorem we get that $\lim _{k \rightarrow \infty} H_{k}(w)=H_{0}(w)$ is a harmonic function, or $H_{0}(w)$

* Project supported by the National Natural Science Foundation of China.

Received October 25, 1991. 
$=\infty$.

Because $\left\{R_{k}\right\}$ is an exhaustion of $R$, there is a $k \geqq 1$ such that $w \in R_{k}$ for given $w \in R$, then $H_{0}(w) \geqq H_{k}(w) \geqq|F(w)|^{p}$. So $H_{0}(w)$ is a harmonic majorant of $|F(w)|^{p}$ on $R$.

In the next step we will prove that $H_{0}(w)$ is the least harmonic majorant of $|F(w)|^{p}$ on $R$, i.e., if $\widetilde{H}(w)$ is another harmonic majorant of $|F(w)|^{p}$ on $R$, we must show that $\tilde{H}(w) \geqq H_{0}(w)$ for each $w \in R$.

Suppose there is a $\zeta \in R$ such that $\tilde{H}(\zeta)<H_{0}(\zeta)$. Because $\left\{R_{k}\right\}$ is the exhaustion of $R$ we know there is a $k_{1}>0$ such that $\zeta \in R_{k_{1}}$. Because $H_{0}(\zeta)=\lim _{k \rightarrow \infty} H_{k}(\zeta)$, there is a $k_{2} \geqq 1$ such that $\tilde{H}(\zeta)<H_{k_{2}}(\zeta)$. Taking $k_{0}=\max \left(k_{1}, k_{2}\right)$, then $\zeta \in R_{k_{1}} \subset$ $R_{k_{0}}$ and $\tilde{H}(\zeta)<H_{k_{2}}(\zeta) \leqq H_{k_{0}}(\zeta)$. Thus $\widetilde{H}(w)$ is a harmonic majorant of $|F(w)|^{p}$ on $R_{k_{0}}$ and $\tilde{H}(\zeta)<H_{k_{0}}(\zeta)$ on $\zeta \in R_{k_{0}}$. This conclusion contradicts to the fact that $H_{k_{0}}(w)$ is the least harmonic majorant of $|F(w)|^{p}$ on $R_{k_{0}}$.

Thus $H_{0}(w)$ is the least harmonic majorant of $|F(w)|^{p}$ on $R$, i.e., $H(w) \equiv$ $H_{0}(w)=\sup _{k \geqq 1} H_{k}(w)=\lim _{k \rightarrow \infty} H_{k}(w)$. This completes the proof.

For the BMOA on the above Riemann surfaces, T.A. Metzger has given the definition as follows [6]: Let $F$ be an analytic function on $R$. We say $F \in B M O A(R)$ if

$$
B_{R}^{2}(F)=\frac{2}{\pi} \sup _{a \in R} \iint_{R}\left|F^{\prime}(w)\right|^{2} G_{R}(w, a) d w d \bar{w}<\infty .
$$

We have the next lemma :

LEMMA 2.2. Let $R_{1} \subset R_{2} \subset \cdots \subset R_{k} \rightarrow R$ be an exhaustion of $R$, where $R_{k}$ are compact bordered Riemann surfaces, then

$$
B_{R_{k}}(F) \leqq B_{R}(F) \text {. }
$$

Proof. Let $G_{k}(w, a)$ be the Green's function on $R$ with logarithmic singularity at $a \in R_{k}$. Let

$$
G(w)=G_{R}(w, a)-G_{k}(w, a) .
$$

It is easy to verify that $G(w)$ is a harmonic function on $R_{k}$, and $\left.G(w)\right|_{\partial R_{k}}=$ $\left.G_{R}(w, a)\right|_{\partial R_{k}}>0$. Using the maximum principle of harmonic function we have $G(w)>0$ for each $w \in R_{k}$, i.e.,

Thus

$$
G_{k}(w, a)<G_{R}(w, a) .
$$

$$
\iint_{R_{k}}\left|F^{\prime}(w)\right|^{2} G_{k}(w, a) d w d \bar{w} \leqq \iint_{R}\left|F^{\prime}(w)\right|^{2} G_{R}(w, a) d w d \bar{w} .
$$

Taking supremum we have

$$
B_{R_{k}}^{2}(F) \leqq B_{R}^{2}(F),
$$

which is the conclusion of the lemma. 
We will give an equivalent definition of BMOA on Riemann surfaces by the exhaustion process in the following theorem.

THEOREM 2.3. Let $R$ be a Riemann surface which possesses a Green's functıon. For each $a \in R$ we possess an exhaustion $R_{a, 1} \subset R_{a, 2} \subset \cdots \subset R_{a, k} \rightarrow R$ such that $a \in R_{a, 1}$. Where $R_{a, k}$ are compact bordered Riemann surfaces, $1 \leqq k<\infty$. $F$ is an analytic function on $R$. Thus we have

$$
B_{R}^{2}(F)=\sup _{a \in R} \sup _{k \geqq 1} H_{a, k}(a),
$$

where $H_{a, k}(w)$ is the least harmonic majorant of $|F(w)-F(a)|^{2}$ on $R_{a, k}$.

Proof. Suppoae $H_{a}(w)$ be the least harmonic majorant of $|F(w)-F(a)|^{2}$ on $R$. From lemma 2.1 we have $H_{a}(w)=\lim _{k \rightarrow \infty} H_{a, k}(w)$. So $H_{a}(a)=\lim _{k \rightarrow \infty} H_{a, k}(a)=$ $\sup _{k \geqq 1} H_{a, k}(a)$. Following lemma 1 of [5] we have

$$
B_{R}^{2}(F)=\sup _{a \in R} H_{a}(a) .
$$

Thus

$$
B_{R}^{2}(F)=\sup _{a \in R} \sup _{k \geqq 1} H_{a, k}(a) .
$$

The proof of theorem 2.3 is completed.

As an application of lemma 2.1 we can give an equivalent definition of $V M O A$ on Riemann surfaces. Firstly let us recall the definition of $V M O A(R)$. Let $R$ be a Riemann surface which possesses a Green's function $G_{R}(w, a)$. $F$ is analytic on $R$. We say $F \in V M O A(R)$ if

$$
\lim _{a \rightarrow \partial R} \iint_{R}\left|F^{\prime}(w)\right|^{2} G_{R}(w, a) d w d \bar{w}=0,
$$

where $\partial R$ is the ideal border of $R$.

Using lemma 2.1 and the lemma 1 of [5] we have the following conclusion immediately :

PROPOSITION 2.4. Let $F$ be an analytic function on Riemann surface $R$, then $F \in V M O A(R)$ if and only if

$$
\lim _{a \rightarrow \partial R} \lim _{k \rightarrow \infty} H_{a, k}(a)=0 .
$$

The meaning of $H_{a, k}(w)$ is the same as that of theorem 2.3.

Next we will give a John-Nirenberg type theorem for BMOA on Riemann surfaces.

THeorem 2.5. Let $R$ be a Riemann surface which possesses a Green's function $G_{R}(w, a)$. For each $a \in R$ we possess an exhaustion $R_{a, 1} \subset R_{a, 2} \subset \cdots \subset R_{a, k} \rightarrow R$, where $R_{a, k}(1 \leqq k<\infty)$ are compact bordered Riemann surfaces and $a \in R_{a, 1}$. $F$ is an analytic function on $R$. Then $F \in B M O A(R)$ if and only if for every $k \geqq 1$ 
and every $a \in R$,

$$
\int_{E_{a, k, \lambda}} \frac{\partial G_{k}(w, a)}{\partial n} d s \leqq K e^{-\beta \lambda} .
$$

Where $E_{a, k, \lambda}=\left\{z \in \partial R_{a, k},|F(w)-F(a)|>\lambda\right\} . \partial / \partial n$ is the inner normal derivative with respect to $R_{a, k}$. K is an absolute constant. When $F \in B M O A(R), \beta=$ $c / B_{R}(F), c$ is another absolute constant.

Proof. Suppose $F \in B M O A(R)$. For $a \in R$ and the exhaustion $a \in R_{a, 1} \subset$ $R_{a, 2} \subset \cdots \subset R_{a, k} \rightarrow R$, because $R_{a, k}$ are compact bordered Riemann surfaces, by lemma 2.2 we know $F \in B M O A\left(R_{a, k}\right)$. From theorem 1 of [4] we know for every $k \geqq 1$ and every $a \in R$,

$$
\int_{E_{a, k, \lambda}} \frac{\partial G_{k}(w, a)}{\partial n} d s \leqq K e^{-\beta_{k} \lambda},
$$

where $\beta_{k}=c / B_{R_{a, k}}(F)$. From lemma 2.2 we know $B_{R_{a, k}}(F) \leqq B_{R}(F)$. So $\beta_{k} \geqq$ $c / B_{R}(F)=\beta$, therefore $e^{-\beta_{k} \lambda} \leqq e^{-\beta \lambda}$, so we have

$$
\int_{E_{a, k, \lambda}} \frac{\partial G_{k}(w, a)}{\partial n} d s \leqq K e^{-\beta \lambda} .
$$

To see the converse we suppose that for every $a \in R$ and every $k \geqq 1(2.1)$ is true. Let

$$
\Lambda_{a, k}(\lambda)=\int_{E_{a, k, \lambda}} \frac{\partial G_{k}(w, a)}{\partial n} d s,
$$

then if $H_{a, k}(w)$ is the least harmonic majorant of $|F(w)-F(a)|^{2}$ on $R_{a, k}$, we have

$$
\begin{aligned}
H_{a, k}(w) & =\int_{\partial R_{a, k}}|F(w)-F(a)|^{2} \frac{\partial G_{k}(w, a)}{\partial n} d s \\
& =2 \int_{0}^{\infty} \lambda \Lambda(\lambda) d \lambda \leqq 2 K \int_{0}^{\infty} \lambda e^{-\beta \lambda} d \lambda \\
& =\frac{2 K}{\beta^{2}}<\infty .
\end{aligned}
$$

so

$$
\sup _{a \in R} \sup _{k \geq 1} H_{a, k}(w) \leqq \frac{2 K}{\beta^{2}}<\infty .
$$

From theorem 2.3 we have $F \in B M O A(R)$ and the proof is completed.

It is analogous to the corollary of [4], using theorem 2.3 and theorem 2.5, noting the next equation [8]:

$$
\int_{\partial R_{a, k}} \frac{\partial G_{k}(w, a)}{\partial n} d s=2 \pi,
$$


we have the corollary as follows:

COROLlaRY 2.6. Let $R$ be a Riemann surface which possesses a Green's function. For each $a \in R$ we possess an exhaustion $R_{a, 1} \subset R_{a, 2} \subset \cdots \subset R_{a, k} \rightarrow R$, where $R_{a, k}(1 \leqq k<\infty)$ are compact bordered Riemann surfaces and $a \in R_{a, 1}$. Thus $F \in B M O A(R)$ if and only if

$$
\sup _{a \in R} \sup _{k \geqq 1} \int_{\partial R_{a, k}}|F(w)-F(a)|^{p} \frac{\partial G_{k}(w, a)}{\partial n} d s=M_{p}<\infty, \quad 1 \leqq p<\infty .
$$

and $M_{p} \sim B_{R}^{p}(F)$. When $F \in B M O A(R), M_{p} \leqq\left(K / C^{p}\right) \Gamma(p+1)\left(B_{R}(F)\right)^{p}$.

\section{BMOA on regular Riemann surfaces.}

Let $R$ be a Riemann surface. $R \notin O_{G}$. We call $R$ be regular if for each $w \in R, \lim _{a \rightarrow \partial R} G_{R}(w, a)=0$. A simple example of regular Riemann surface is the unit disk. For the regular Riemann surface $R$, John-Nirenberg type theorem for $B M O A(R)$ has a much simple form. We firstly give two lemmas:

LEMmA 3.1. [1] If $R$ is a regular Riemann surface. Then for a given compact set $R_{0} \subset R$ and $\varepsilon>0$, there is a compact set $S_{0} \subset R$ such that $w \in R \backslash S_{0}$ implies $G_{R}(w, a)<\varepsilon$ for every $a \in R_{0}$. Especıally for each $a \in R$, we can take $R_{0}=\{a\}$.

From lemma 3.1 it is easy to know that if $R$ is a regular Riemann surface, then for every $a \in R$ and every $t>0 \Gamma_{a, t}=\left\{w \in R, G_{R}(w, a)=t\right\}$ is constructed by a finite number of analytic Jordan curves.

LEMMA 3.2. Let $R$ be a regular Riemann surface. For every $a \in R$ and every $t>0, \quad R_{a, t}=\left\{w \in R, G_{R}(w, a) \geqq t\right\}$ is a compact bordered Riemann surface whose border is $\Gamma_{a, t}$. $G_{R_{a, t}}(w, a)=G_{R}(w, a)-t$ is the Green's function on $R_{a, t}$ with logarithmic singularity at $a$, and $\left\{R_{a, t}\right\}$ is an exhaustion of $R$ when $t$ decreases to zero.

Proof. Fix $a \in R$. Suppose $\left\{w_{n}\right\} \subset R_{a, t}, w_{n} \rightarrow w_{0}$. From lemma 3.1, there is a compact set $S_{0} \subset R$ such that every $w \in R \backslash S_{0}$ implies $G_{R}(w, a)<t$. So $R_{a, t} \subset S_{0}$, i.e., $\left\{w_{n}\right\} \subset S_{0}$. Because $S_{0}$ is compact we know $w_{0} \in S_{0} \subset R$, thus $G_{R}\left(w_{0}, a\right)=\lim _{n \rightarrow \infty} G_{R}\left(w_{n}, a\right) \geqq t$. Equivalently, $w_{0} \in R_{a, t}$. So $R_{a, t}$ is a closed subdomain of the compact set $S_{0} \subset R$, then we have $R_{a, t}$ is a compact domain.

Let $w \in R$ such that $G_{R}(w, a)>t>0$. From the continuity of $G_{R}(w, a)$ we know there is a sufficient small parametric disk $U_{\varepsilon}$ which contains $w$ such that every $\zeta \in U_{\varepsilon}$ implies $G_{R}(\zeta, a)>t$. Thus $U_{\varepsilon} \subset R_{a, t}$. So $w$ can not be the border point of $R_{a, t}$, and the border of $R_{a, t}$ is constructed by $\Gamma_{a, t}$, i.e., $R_{a, t}$ is a compact bordered Riemann surface.

By direct verification we can know $G_{R_{a, t}}(w, a)=G_{R}(w, a)-t$ is the Green's 
function of $R_{a, t}$ with logarithmic singularity at $a$.

For each $w \in R$, there is a $t_{1}>0$ such that $G_{R}(w, a)>t_{1}>0$. So $w \in R_{a, t_{1}}$, and we conclude that $\left\{R_{a, t}\right\}$ is an exhaustion of $R$ when $t$ decreases to zero. The proof is completed.

From lemma 3.2, for the regular Riemann surface $R$, we have

$$
\frac{\partial G_{R_{a, t}}(w, a)}{\partial n}=\frac{\partial G_{R}(w, a)}{\partial n} .
$$

Where $\partial / \partial n$ is the inner normal derivative with respect to the compact bordered Riemann surface $R_{a, t}$.

From (3.1), we can conclude immediately that for every $a \in R$ and every $t>0$,

$$
\int_{\Gamma_{a, t}} \frac{\partial G_{R}(w, a)}{\partial n} d s=2 \pi .
$$

Let $F(w)$ be an analytic function on $R . H(w)$ is the least harmonic majorant of $|F(w)|^{p}$, from lemma 2.1 and (3.1) we have

$$
H(a)=\frac{1}{2 \pi} \sup _{t>0} \int_{\Gamma_{a, t}}|F(w)|^{p} \frac{\partial G_{R}(w, a)}{\partial n} d s .
$$

If $|F(w)|^{p}$ has no harmonic majorant on $R$ we denote $H(w)=\infty$.

From this, corresponding to theorem 2.3 and noting (3.3) we have

THEOREM 3.3. Let $F$ be an analytic function on a regular Riemann surface $R$, then

$$
B_{R}^{2}(F)=\frac{1}{2 \pi} \sup _{a \in R} \sup _{t>0} \int_{\Gamma_{a, t}}|F(w)-F(a)|^{2} \frac{\partial G_{R}(w, a)}{\partial n} d s .
$$

Corresponding to proposition 2.4 we have

PROPOSITION 3.4. Let $F$ be an analytıc function on a regular Riemann surface $R$, then $F \in V M O A(R)$ if and only if

$$
\lim _{a \rightarrow \partial R} \lim _{t \rightarrow 0+} \int_{\Gamma_{a, t}}|F(w)-F(a)|^{2} \frac{\partial G_{R}(w, a)}{\partial n} d s=0 .
$$

From theorem 2.5 we can easily deduce John-Nirenberg type theorem on regular Riemann surfaces. It has a much simple form.

THEOREM 3.5. Let $R$ be a regular Riemann surface. $F$ is an analytic function on $R$. Then $F \in B M O A(R)$ if and only if for every $a \in R$ and every $t>0$

$$
\int_{E_{a, t, \lambda}} \frac{\partial G_{R}(w, a)}{\partial n} d s \leqq K e^{-\beta \lambda} .
$$

Where $E_{a, t, \lambda}=\left\{w \in \Gamma_{a, t},|F(w)-F(a)|>\lambda\right\} . \partial / \partial n$ is the inner normal derivative with respect to $R_{a, t} . K$ is an absolute constant. When $F \in B M O A(R), \beta=$ 
$c / B_{R}(F), c$ is another constant.

Corresponding to corollary 2.6 we have

COROLLARY 3.6. Let $R$ be a regular Riemann surface. $F$ is an analytic function on $R . \quad F \subseteq B M O A(R)$ if and only if

$$
\sup _{a \in R} \sup _{t>0} \int_{\Gamma_{a, t}}|F(w)-F(a)|^{p} \frac{\partial G_{R}(w, a)}{\partial n} d s=M_{p}<\infty, \quad 1 \leqq p<\infty,
$$

and $M_{p} \sim B_{R}^{p}(F)$, when $F \in B M O A(R), M_{p} \leqq\left(K / C^{p}\right) \Gamma(p+1)\left(B_{R}(F)\right)^{p}$.

As an application of theorem 3.5, we point out that the distribuion function on some area measure of $B M O A$ on the regular Riemann surface $R$ has exponential decay.

Let $G_{R}^{*}(w, a)$ be the conjugate function of $G_{R}(w, a)$, then $P(w)=G_{R}(w, a)+$ $\imath G_{R}^{*}(w, a)$ is an analytic function on $R \backslash\{a\}$. We have

COROLlary 3.7. Let $R$ be a regular Riemann surface. If $F \in B M O A(R)$, then

$$
\iint_{D_{a, \lambda}}\left|P^{\prime}(w)\right|^{2} e^{-k G_{R}(w, a)} d w d \bar{w} \leqq \frac{K}{k} e^{-\beta \lambda} .
$$

where $k$ is an arbitrary positive integer. $\beta=c / B_{R}(F), K, c$ are absolute constants. $D_{a, \lambda}=\{w \in R,|F(w)-F(a)|>\lambda\}$.

Proof. Because $G_{R}(w, a)$ is a constant on $\Gamma_{a, t}$, we have

$$
i P^{\prime}(w) d w=-\frac{\partial G_{R}^{*}(w, a)}{\partial s} d s=\frac{\partial G_{R}(w, a)}{\partial n} d s
$$

along $\Gamma_{a, t}$, where ds is the arc length and $\partial / \partial n$ is the inner normal derivative with respect to $R_{a, t}$. Thus we have

$$
\left|P^{\prime}(w)\right|^{2}=\left(\frac{\partial G_{R}(w, a)}{\partial n}\right)^{2}
$$

along $\Gamma_{a, t}$. Because $G_{R}(w, a)=t$ along $\Gamma_{a, t}$ we have $\left(\partial G_{R}(w, a) / \partial n\right) d n=d t$. Thus

$$
\begin{aligned}
& \iint_{D_{a, \lambda}}\left|P^{\prime}(w)\right|^{2} e^{-k G_{R}(w, a)} d w d \bar{w} \\
= & \iint_{D_{a, \lambda}}\left(\frac{\partial G_{R}(w, a)}{\partial n}\right)^{2} e^{-k G_{R}(w, a)} d s d n \\
= & \int_{0}^{\infty} \int_{E_{a, t, \lambda}} \frac{\partial G_{R}(w, a)}{\partial n} e^{-k t} d s d t \leqq \int_{0}^{\infty} K e^{-\beta \lambda} e^{-k t} d t \\
= & \frac{K}{k} e^{-\beta \lambda .}
\end{aligned}
$$


Where $\beta=c / B_{R}(F), K, c$ are absolute constants, and the proof is completed.

\section{4. $B M O A$ and Ba spaces on Riemann surfaces.}

Ba spaces was introduced by Ding Xiaxi and Luo Peizhu in [2]. In [4], we have discussed a special class of Ba spaces $H^{B a}$ on compact bordered Riemann surfaces and have given a new characteristic of BMOA on compact bordered Riemann surfaces. Next we will show that on the general open Riemann surface $R$ which possesses a Green's function, the above conclusion is still true.

We firstly recall Hardy spaces $H^{p}(R)$ on the Riemann surface $R(1 \leqq p<\infty)$.

$$
\begin{gathered}
H^{p}(R)=\left\{F, F \text { is analytic on } R \text { and }|F(w)|^{p}\right. \text { has a } \\
\text { harmonic majorant on } R\} .
\end{gathered}
$$

We have known [3] if $H(w)$ is the least harmonic majorant of the subharmonic funtion $|F(w)|^{p}$, the norm of $H^{p}(R)$ can be defined by

$$
\|F\|_{p}=|H(a)|^{1 / p}, \quad a \in R .
$$

If we exchange the reference point we get equivalent norms. Notice by lemma 2.1, for an arbitrary exhaustion $R_{1} \subset R_{2} \subset \cdots \subset R_{k} \rightarrow R$, which $a \in R_{1}$, we have

$$
\|F\|_{p}^{p}=\lim _{k \rightarrow \infty}\left|H_{k}(a)\right|=\sup _{k \geqq 1}\left|H_{k}(a)\right|,
$$

where $H_{k}(w)$ is the least harmonic majorant of $|F(w)|^{p}$ on $R_{k}$.

Now let $E(\zeta)=\sum_{m=1}^{\infty} a_{m} \zeta^{m}$ be a finite order $(\rho<\infty)$ and mean type $(\sigma<\infty)$ entire function, and $a_{m} \geqq 0$. The sequence $\left\{p_{m}\right\}$ has the property $1 \leqq p_{1} \leqq p_{2} \leqq$ $\cdots \leqq p_{m} \rightarrow \infty$ and

$$
\varlimsup_{m \rightarrow \infty} \frac{p_{m}}{m^{1 / p}}=p^{*}<\infty
$$

For $F(w) \in \bigcap_{m=1}^{\infty} H^{p_{m}}(R)$ we set

$$
I(F, \alpha)=\sum_{m=1}^{\infty} a_{m}\|F\|_{p_{m}}^{m} \alpha^{m},
$$

and use $d_{F}$ to denote the convergence radius of (4.3). Define

$$
H^{B a}(R)=\left\{F, F \in \bigcap_{m=1}^{\infty} H^{p_{m}}(R), \text { and } d_{F}>0\right\} .
$$

The norm of $F$ in $H^{B a}(R)$ is defined by

$$
\|F\|_{B a}=\inf \left\{\frac{1}{|\alpha|}, I(F,|\alpha|) \leqq 1\right\} .
$$

Set 


$$
\|F\| \mid=\sup _{a \in R}\left\{\|F(w)-F(a)\|_{B a}\right\} .
$$

Thus we have

THEOREM 4.1. Let $F$ be an analytic function on the Riemann surface $R$, then there is a constant $c$ such that

$$
c^{-1} B_{R}(F) \leqq|\|F\|| \leqq c B_{R}(F) .
$$

The proof of this theorem is similar to that of theorem 2 in [4], so we omit it here.

Acknowledgment. The author wishes to thank Professors He Yuzan and Ouyang Caiheng for their enthusiastic guidance and the referee for several useful suggestions.

\section{REFERENCES}

[1] R. Aulaskari, On VMOA for Riemann surfaces, Can. J. Math., 40 (1988), $1174-1185$.

[2] Ding Xiaxi and Luo Peizhu, Ba spaces and some estimates of Laplace operator, J. Sys. Sci. Math. Sci., 1 (1981), 9-33.

[3] M. Heins, Hardy classes on Riemann surfaces, Lecture Notes in math., 98, Springer- Verlag, Berlin, 1969.

[4] He Yuzan and Zhao Ruhan, BMOA and Ba spaces on compact bordered Riemann surfaces, Chinese Science Bulletin, 36 (1991), 20, 1677-1682.

[5] S. KobayAshi, Range sets and BMO norms of analytic functions, Can. J. Math., 36 (1984), 745-755.

[6] T.A. Metzger, On BMOA for Riemann surfaces, Can. J. Math., 33 (1981), $1255-1260$.

[7] T.A. Metzger, Bounded mean oscillation and Riemann surfaces, BMO in Complex Analysis, Joensuu, 1989, 79-99.

[8] M. TsujI, Potential Theory in Modern Function Theory (Maruzen Co. Ltd., Tokyo, 1959).

Wuhan Institute of Mathematical

Sciences, Academia Sinica

P.O.BOX 71007 WUHAN,

430071 P.R. CHINA 